\title{
ASK1/p38-mediated NLRP3 inflammasome signaling pathway contributes to aberrant retinal angiogenesis in diabetic retinopathy
}

\author{
WENJUN ZOU ${ }^{1,2^{*}}$, SHASHA LUO ${ }^{1,2^{*}}$, ZHENGWEI ZHANG $^{1,2}$, LIBO CHENG $^{1,2}$, XIAOLI HUANG ${ }^{1,2}$, \\ NANNAN DING ${ }^{1,2}$, YING PAN ${ }^{1,2}$ and ZHIFENG WU ${ }^{1,2}$ \\ ${ }^{1}$ Department of Ophthalmology, The Affiliated Wuxi No. 2 People's Hospital of Nanjing Medical University; \\ ${ }^{2}$ Department of Ophthalmology, Affiliated Wuxi Clinical College of Nantong University, Wuxi, Jiangsu 214002, P.R. China
}

Received May 27, 2020; Accepted November 18, 2020

DOI: $10.3892 /$ ijmm.2020.4833

\begin{abstract}
Diabetic retinopathy (DR) is the leading cause of blindness among the working-age population in several countries. Despite the available treatments, some patients are diagnosed at the late stages of the disease when treatment is more difficult. Hence, it is crucial that novel targets are identified in order to improve the clinical therapy of DR. In the present study, an animal model of DR and a cell model using primary human retinal microvascular endothelial cells exposed to high glucose were constructed to examine the association between apoptosis signal-regulating kinase 1 (ASK1)/p38 and NLR family pyrin domain containing 3 (NLRP3) in DR. The results revealed that DR induced inflammatory response and microvascular cell proliferation. NLRP3 contributed to DR-mediated inflammatory development and progression, which promoted the expression of inflammatory-related cytokines. In addition, NLRP3 promoted the tube formation of retinal microvascular endothelial cells and angiogenesis. Moreover, further research indicated that the NLRP3-mediated aberrant retinal angiogenesis in DR was regulated by ASK1 and p38. It was thus suggested that ASK1/p38 may be novel target for the treatment of DR.
\end{abstract}

\section{Introduction}

Diabetic retinopathy (DR) is a common and specific microvascular complication of diabetes, and it remains the leading

Correspondence to: Dr Wenjun Zou or Dr Zhifeng Wu, Department of Ophthalmology, The Affiliated Wuxi No. 2 People's Hospital of Nanjing Medical University, 68 Zhongshan Road, Wuxi, Jiangsu 214002, P.R. China

E-mail: wendyzwj0805@njmu.edu.cn

E-mail: zhifengwu2018@126.com

*Contributed equally

Key words: diabetic retinopathy, inflammatory, ASK1/p38, NLRP3, angiogenesis cause of preventable blindness among the working-age population $(1,2)$. It is associated with diabetes and increases the risk of life-threating systemic vascular complications, which include stroke, coronary heart disease, and heart failure $(3,4)$. The main reason for the irreversible visual impairment induced by DR is retinal neovascularization (5). DR is usually associated with the disruption of certain signaling pathways and the aberrant expression of functional molecules (6). Hence, the potential molecular mechanisms responsible for retinal neovascularization are of utmost importance. In addition, the development of effective diagnostic and therapeutic strategies for patients with DR is imperative.

Some studies have demonstrated that DR can cause the activation of the polyol pathway and hexamine pathways, the accumulation of advanced glycation end products, inflammation and protein kinase $\mathrm{C}$ activation $(5,7,8)$. There is increasing evidence to indicate that the inflammatory response plays an important role in the pathogenesis of DR (7). Moreover, the high expression levels of pro-inflammatory cytokines are observed in retinas from animals with diabetes (5). Therefore, the inhibition of inflammatory signaling may become an effective treatment strategy for DR.

The inflammasome is a multiprotein scaffolding complex which includes a member of the NOD-like receptor family, pyrin domain containing family member (NLRP), procaspase 1 and apoptosis-associated speck-like protein containing a CARD (9). To date, the NLR family pyrin domain containing 3 (NLRP3) inflammasome is known to lead to the secretion of the pro-inflammatory cytokine, IL- $1 \beta$, by recognizing danger signals and apoptosis-associated speck-like protein to activate caspase-1 (9). Related research has revealed that NLRP3 activation plays a crucial role in metabolic disease, such as type 2 diabetes (10). Moreover, vitreous clinical samples from patients with various stages of diabetes have been revealed to exhibit an increased expression of the NLRP3 and related inflammatory proteins, with the greatest increase observed in patients with proliferative DR (11). Thus, it appears that the NLRP3 inflammasome may be involved in retinal disease.

Apoptosis signal-regulating kinase 1 (ASK1), a member of the mitogen-activated protein kinase kinase kinase (MAP3K) family, is an important stress responsive protein kinase which 
plays a crucial role in the initiation of numerous diseases, including neurodegenerative, cardiovascular, inflammatory, autoimmunity, and metabolic disorders $(12,13)$. The MAPK member, p38, is a serine/threonine protein kinase, which responds to several cellular processes and external stress signaling, such as cell differentiation, cell proliferation, inflammation regulation and cell death $(14,15)$. ASK 1 is the most well-studied family member and is an upstream kinase of the JNK and p38 pathways $(16,17)$. It has been demonstrated that ASK1 is activated in response to a variety of stress-related stimuli via distinct mechanisms and activates MKK4 and MKK3, which in turn activate JNK and p38 (18). Related research has revealed that the ASK1/2 signaling complex contributes to pyroptotic cell death by regulating the NLRP3 inflammasome (19). Although ASK1/p38 plays a substantial role in inflammation, its role in the pathogenesis of DR has yet not been described, at least to the best of our knowledge.

In the present study, an animal model of DR and a cell model using primary human retinal microvascular endothelial cells (HRMECs) exposed to high glucose (HG) were constructed to investigate the association between ASK1/p38 and NLRP3 in DR.

\section{Materials and methods}

Cell culture and stimulation. The HRMECs were purchased from Cell Systems, and were routinely cultured in M199 medium (EMD Millipore) supplemented with 100 units of penicillin and $100 \mu \mathrm{g}$ of streptomycin (both from Sigma-Aldrich; Merck KGaA) per milliliter of medium. All cells (passages 5-12) were cultured in grade plastic-ware and maintained in an atmosphere of $5 \% \mathrm{CO}_{2}$ at $37^{\circ} \mathrm{C}$.

For the establishment of the HG cell models, the HRMECs were cultured in conditioned medium with $5 \mathrm{mM}$ [serving as the normal glucose (NG) group] or $30 \mathrm{mM}$ (the HG group) D-glucose (Sigma-Aldrich; Merck KGaA) and incubated at $37^{\circ} \mathrm{C}$ with $5 \% \mathrm{CO}_{2}$; the $\mathrm{HG}$ group was then treated with or without $1 \mu \mathrm{M}$ of the NLRP3 inhibitor, CY-09, $1 \mu \mathrm{M}$ of the ASK1 inhibitor, GS-444217 or $10 \mu \mathrm{M}$ of the p38 inhibitor, SB203580 (all from MedChemExpress) for $24 \mathrm{~h}$, respectively. Each inhibitor was dissolved in dimethyl sulfoxide (DMSO) to a concentration of $50 \mathrm{mM}$ for use as stock solutions that were diluted to the required concentrations for in vitro experiments.

Establishment and treatment of the animal model of DR. Male C57/BL/J mice ( $\mathrm{n}=30 ; 10$ weeks old; 19-21 g) were housed under specific pathogen-free conditions at $25^{\circ} \mathrm{C}$ with a 12-h light/dark cycle and free access to food and water at the Animal Research Center of the Affiliated Wuxi No. 2 People's Hospital of Nanjing Medical University, which observed animal growth status every day. All of the animal experiments were performed in accordance with the Guidelines for the Care and Use of Laboratory Animals published by the US National Institutes of Health (NIH Publication. no. 85-23, revised 1996), and were approved by the Experimental Animal Ethics Committee of the Affiliated Wuxi No. 2 People's Hospital of Nanjing Medical University (Nanjing, China). Streptozotocin (STZ)-induced hyperglycemic mice were utilized as the type I diabetic-like model associated with retinopathy, as previously described (20). Male $\mathrm{C} 57 / \mathrm{BL} / \mathrm{J}$ mice received constitutive intraperitoneal injections of $50 \mathrm{mg} / \mathrm{kg} \mathrm{STZ}$ in a citric buffer $(\mathrm{pH} \mathrm{4.5)}$ once/day for 5 days. After the final injection, the 4-h fasting blood glucose levels were determined, which were between 15.0 to $20.0 \mathrm{nmol} / \mathrm{l}$. The mice in the control group were injected only with citric buffer. The hyperglycemic (HG) mice were randomly divided into 5 groups as follows: The normal group (NG), the HG-treated group, the $\mathrm{HG}+$ the NLRP3 inhibitor CY-09 treatment group, the HG + ASK1 inhibitor GS-444217 treatment group and the $\mathrm{HG}+\mathrm{p} 38$ inhibitor SB203580 treatment group. NLRP3 inhibitor CY-09 was administered orally to the C57BL/6J mice at a dose of $2.5 \mathrm{mg} / \mathrm{kg}$ once a day for 6 weeks. ASK1 inhibitor GS-444217 was administered orally to the mice at a dose of $10 \mathrm{mg} / \mathrm{kg}$ once a day for 6 weeks. The p38 inhibitor SB203580 was administered orally to the mice at a dose of $15 \mathrm{mg} / \mathrm{kg}$ once a day for 6 weeks. The mice in the normal and model groups received the same volume of water. All inhibitors were first dissolved in a small quantity of DMSO, and then evenly mixed with $0.5 \% \mathrm{CMC}-\mathrm{Na}$ solution. The final concentration of DMSO was $5 \%$. The treatment period lasted for 6 weeks. Twenty-four hours after the last treatment, the mice from all of the groups were sacrificed by cervical dislocation. When the breathing of the mouse stopped, the pupils were dilated, and the heartbeat stopped, the mice were considered euthanized. Then, the eyeballs were collected from the sacrificed mice. Some of the retinas were enucleated and placed in $4 \%$ paraformaldehyde overnight for use in immunofluorescence staining. The other retinas were stored at $-80^{\circ} \mathrm{C}$ for use in RT-qPCR or western blot analysis. No anesthetics were used in this model.

Reverse transcription-quantitative PCR (RT-qPCR). Total RNA was isolated from the HRMECs and tissue samples using TRIzol reagent (Sigma-Aldrich; Merck KGaA) and was reverse transcribed into cDNA using Reverse Transcription Kit (Bio-Rad Laboratories, Inc.) according to the manufacturer's instructions. RT-qPCR was then performed on a MyiQ single-color RT-PCR detection system with SYBR-Green Supermix (Vazyme Biotech Co., Ltd.). The qPCR thermocycling conditions were as follows: $95^{\circ} \mathrm{C}$ for $3 \mathrm{~min}, 40$ cycles at $95^{\circ} \mathrm{C}$ for $30 \mathrm{sec}$, annealing at $60^{\circ} \mathrm{C}$ for $45 \mathrm{sec}$, and a final elongation step at $72^{\circ} \mathrm{C}$ for $20 \mathrm{sec}$. The CFX96 Real-Time PCR System (Bio-Rad Laboratories, Inc.) was used to conduct the reaction and detection. The primer sequences for each gene were as follows: IL-6 forward, 5'-CTTCACAAGTCGGAGGCTTAAT-3' and reverse, 5'-GCATCATCGCTGTTCATACAATC-3'; TNF- $\alpha$ forward, 5'-GCCTCAGCCTCTTCTCATTC-3' and reverse, 5'-GGGAACTTCTCCTCCTTGTTG-3'; IL-1 $\beta$ forward, 5'-TGACCCATGTGAGCTGAAAG-3' and reverse, 5'-CGT TGCTTGTCTCTCCTTGTA-3'; GAPDH forward, 5'-GGG AAACCCATCACCATCTT-3' and reverse, 5'-CCAGTA GACTCCACGACATACT-3'. Relative mRNA expression was normalized to that of GAPDH and was determined using the comparative $\mathrm{Cq}$ method $\left(2^{-\Delta \Delta \mathrm{Cq}}\right)$ (21).

Western blot analysis. For western blot analysis, the protein from the HRMECs and tissue samples was lysed in RIPA 
buffer containing protease inhibitor cocktail [Generay Biotech (Shanghai) Co., Ltd.]. Protein concentration was determined using a BCA assay kit (cat. no. 23250; Pierce; Thermo Fisher Scientific, Inc.). Equal amounts (30 $\mu \mathrm{g}$ each) of total proteins were subjected to electrophoresis on sodium dodecyl sulphate polyacrylamide $10 \%$ gels and transferred onto nitrocellulose membranes (Life Technologies; Thermo Fisher Scientific, Inc.). After blocking using 5\% BSA (Sigma-Aldrich; Merck KGaA) at room temperature for $2 \mathrm{~h}$, the NLRP3, ASK1, p38, interleukin (IL)-6, tumor necrosis factor (TNF)- $\alpha$, vascular endothelial growth factor (VEGF), IL-1 $\beta$ and GAPDH levels were probed with anti-NLRP3 (product code ab263899), anti-ASK1 (product code ab45178), anti-p38 (product code ab178867), anti-IL-6 (product code ab229381), anti-TNF- $\alpha$ (product code ab215188), anti-VEGF (product code ab53465), anti-IL-1 $\beta$ (product code ab234437) and anti-GAPDH (product code ab8245) antibodies overnight at $4^{\circ} \mathrm{C}$ (all 1:1,000; Abcam). The membranes were then incubated with appropriate secondary antibodies (goat anti-mouse IgG H\&L, 1:10,000, product code ab150115; and goat anti-rabbit IgG H\&L, 1:10,000, product code ab150077; both from Abcam) for $2 \mathrm{~h}$ at room temperature. The blots were detected using Bio-Imaging System and Quality One 1-D analysis software (Bio-Rad Laboratories, Inc.).

Immunofluorescence staining. The sections from the mouse retinal tissues were fixed with ice-cold acetone for $20 \mathrm{~min}$. The slides were blocked with 5\% BSA in PBS for $1 \mathrm{~h}$ at room temperature and subjected to incubation at $4^{\circ} \mathrm{C}$ overnight with the following primary antibody mixtures: Biotin-anti-mouse CD31 (1:100; product code ab222783; from Abcam) or biotin-anti-mouse IB4 (1:100; product code I21411; from Thermo Fisher Scientific, Inc.). The slides were then washed and incubated with streptavidin-Alexa Fluor 488 conjugate (1:200; cat. no. S32354) or streptavidin-Alexa Fluor 594 conjugate (1:200; cat. no. S32356; both from Thermo Fisher Scientific, Inc.) for $90 \mathrm{~min}$ at room temperature. The slides were co-stained with DAPI and mounted with fluoro-gel (Electron Microscopy Science). Confocal images were acquired using a Leica TCS SP5 confocal microscope system (Leica Microsystems GmbH; magnification, x200) and quantified using AxioVision 4.6.3.0 (Carl Zeiss AG).

Analysis of retinal microvascular endothelial cell tube formation. A total of $100 \mu 1$ Matrigel per well was evenly spread on the bottom of a 24 -well plate and allowed to solidify at $37^{\circ} \mathrm{C}$ with $5 \% \mathrm{CO}_{2}$ for $2 \mathrm{~h}$. Differently treated HRMECs, including those in the $\mathrm{NG}$ group, $\mathrm{HG}$ group and $\mathrm{HG}$ group treated with inhibitors, were digested and centrifuged at $1,000 \mathrm{x}$ g for $5 \mathrm{~min}$ at $4^{\circ} \mathrm{C}$; the supernatant was removed and the cells were suspended in complete medium; the cells were separately seeded in 24-well plates at $3 \times 10^{5}$ cells/well and cultured at $37^{\circ} \mathrm{C}$ with $5 \% \mathrm{CO}_{2}$ for $8 \mathrm{~h}$. The tube formation of retinal microvascular endothelial cells was photographed and analyzed using the Image J 1.50 software Angiogenesis Analyzer Plugin (National Institutes of Health).

Measurements of VEGF levels. Retinal tissue from the control group, the HG group and HG-treated with inhibitor groups were homogenized in PBS; the homogenate was centrifuged at $12,000 \times \mathrm{g}$ at $4^{\circ} \mathrm{C}$ for $10 \mathrm{~min}$. Then, the VEGF levels in the supernatant were then examined using the mouse VEGF ELISA kit (cat. no. DVE00; R\&D Systems, Inc.) according to the manufacturer's instruction. For the cell experiment, the cell-cultured supernatants from the different treatment groups were harvested and examined using the mouse VEGF ELISA kit.

Statistical analysis. The data were statistically analyzed using the paired Student's t-test or one-way ANOVA followed by Dunnett's post hoc test with GraphPad Prism 4.0 software (GraphPad Software, Inc.). $\mathrm{P}<0.05$ was considered to indicate a statistically significant difference.

\section{Results}

Inflammatory response and microvascular cell proliferation are induced by DR. It has been demonstrated that DR can activate some signaling pathways and cause inflammatory response (7). Hence, in the present study, the mice with STZ-induced hyperglycemia were utilized as the type I diabetic-like model associated with retinopathy and constructed. The levels of inflammatory cytokines associated with DR were measured; the results revealed that the mRNA expression levels of IL-6, TNF- $\alpha$ and IL-1 $\beta$ were enhanced in the retinas of the mice in the DR groups compared with those of the control group (Fig. 1A). Microvascular formation in the retina was then analyzed. The expression of the vascular marker, CD31, was increased in DR group, as indicated by confocal detection (Fig. 1B). Moreover, the VEGF secretion levels in the DR group were increased compared with those of the control group (Fig. 1C). All of these data indicated that DR induces the inflammatory response and microvascular cell proliferation in the retina.

NLRP3-mediated tissue inflammatory response promotes microvascular cell proliferation in the retina. The analysis of clinical samples from patients with various stages of diabetes has demonstrated that the expression of NLRP3 and related inflammatory proteins is increased in vitreous samples, with the largest levels observed in patients with proliferative DR (10). Hence, in the present study, the role of NLRP3 in microvascular cell proliferation in the retina was investigated. The results revealed that the protein expression of NLRP3 was upregulated in the DR group (Fig. 2A). Then, the DR group was treated with NLRP3 inhibitor; following treatment with the inhibitor, the inflammatory cytokines IL-6, TNF- $\alpha$ and IL- $1 \beta$ mRNA expression levels were decreased compared with the DR group not treated with the inhibitor (Fig. 2B). Moreover, the CD31 expression level was decreased in inhibitor treatment group (Fig. 2C). In addition, the VEGF secretion level was decreased following treatment with NLRP3 inhibitor (Fig. 2D). In order to further investigate the role of NLRP3, the HG cell models using HRMECs were constructed and the cells were treated with or without NLRP3 inhibitor. The results revealed that NLRP3 protein expression levels in the HRMECs in the HG group were upregulated, and were decreased following treatment with the inhibitor (Fig. 2E). In the HRMECs in the HG groups, IL-6, TNF- $\alpha$ and IL-1 $\beta$ mRNA expression levels were 
A

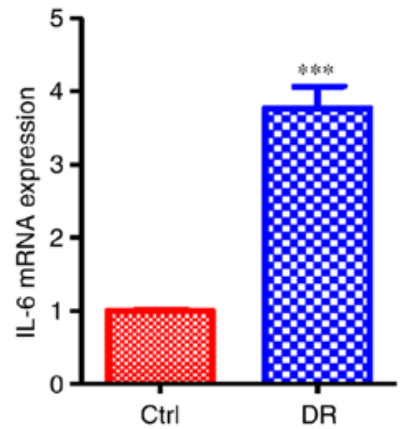

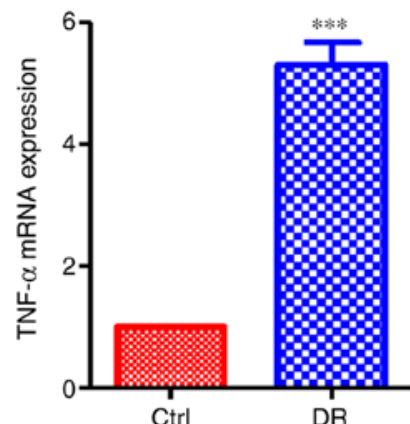

CD31
B

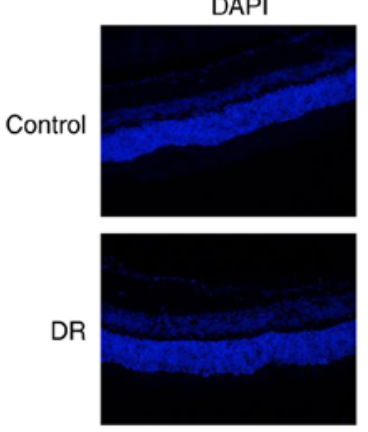

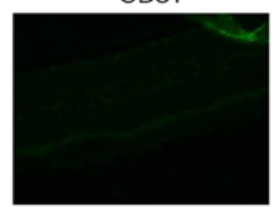

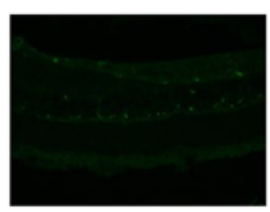

Ctr

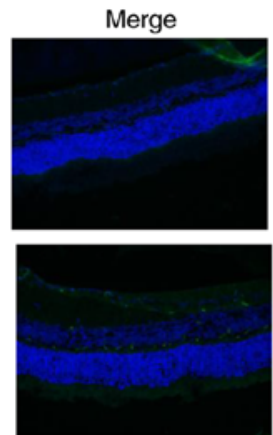

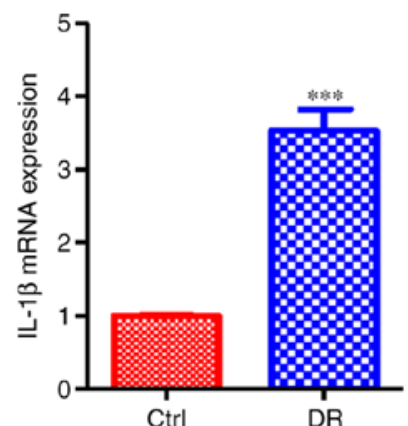

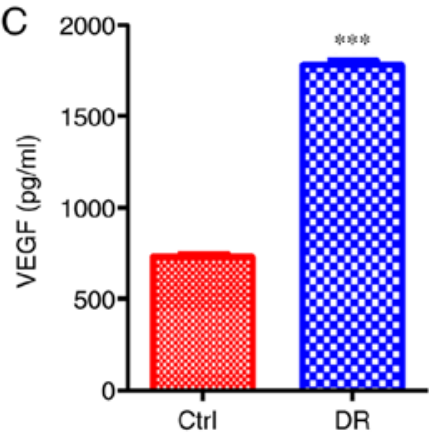

Figure 1. DR induces the inflammatory response and microvascular cell proliferation. (A) mRNA expression levels of the inflammatory-related cytokines, IL-6, TNF- $\alpha$ and IL-1 $\beta$, were enhanced in the DR group. (B) The expression of the vascular marker, CD31, was increased in the DR group, as indicated by confocal analysis. (C) The VEGF secretion levels in the DR group were increased compared with the control group. Values are expressed as the means $\pm \mathrm{SD}\left(\mathrm{n}=6{ }^{*}{ }^{* * *} \mathrm{P}<0.001\right.$ compared with the control). DR, diabetic retinopathy; IL, interleukin; TNF, tumor necrosis factor; VEGF, vascular endothelial growth factor; Ctrl, control.

enhanced compared with the control, and were suppressed by the NLRP3 inhibitor (Fig. 2F). The VEGF secretion levels in the cells were similar to those observed in the animal model of DR (Fig. 2G). Collectively, these data demonstrated that NLRP3-mediated tissue inflammatory response promoted microvascular cell proliferation in retina.

Tube formation of retinal microvascular endothelial cells is inhibited by the blocking of NLRP3. The aforementioned data indicated that NLRP3 was associated with inflammatory response, and promoted the expression of the vascular markers, CD31 and VEGF. Hence, the present study examined the tube formation of retinal microvascular endothelial cells in the retina. As revealed in Fig. 3A, lumen formation was markedly enhanced in HRMECs in the HG group, and was inhibited by the blocking of NLRP3. The tube meshes, nodes and tube length were assessed, and the results revealed that the HRMECs in the HG group formed more meshes and nodes than the controls; moreover, the tube total length in the HG group was higher than that in the control group (Fig. 3B-D). However, after blocking NLRP3, the tube meshes, nodes and tube length were reduced compared with that in the HG group (Fig. 3B-D). These data indicated that the tube formation of retinal microvascular endothelial cells was inhibited by the blocking of NLRP3.

Expression level of the NLRP3 inflammasome is upregulated via the activation of the ASK1/p38 signaling axis. Data have indicated that the NLRP3-induced inflammatory response plays an important role in DR (9). However, the potential mechanisms of the role of NLRP3 in inflammatory response in DR remain unknown. Thus, the present study utilized the
DR model and HG HRMECs model to investigate this matter. The results revealed that the protein expression levels of ASK1 and $\mathrm{p} 38$ were upregulated in the DR and HG HRMECs groups (Fig. 4A and B). Subsequently, in order to investigate whether ASK1 and p38 play a role in the NLRP3-mediated inflammatory response. ASK1 and p38 inhibitors were utilized to block the related protein expression. The data demonstrated that the protein expression levels of NLRP3, inflammatory cytokines (IL-6, TNF- $\alpha$ and IL-1 $\beta$ ) and VEGF were downregulated after the blocking of ASK1 and p38 (Fig. 4C and D). All these data indicated that NLRP3-mediated tissue inflammatory signal response was enhanced through the activation of the ASK1/p38 signaling axis.

Tube formation of retinal microvascular endothelial cells is inhibited via the blocking of the ASK1/p38 signaling axis. NLRP3 plays an important part in the tube formation of retinal microvascular endothelial cells. Moreover, the NLRP3-mediated tissue inflammatory response is enhanced via the activation of the ASK1/p38 signaling axis. Hence, it was hypothesized that the ASK1/p38 signaling axis may mediate the tube formation of retinal microvascular endothelial cells. As revealed in Fig. 5A and C, HG promoted tube formation, and this formation was inhibited by ASK1 and p38 inhibitors. Moreover, the tube meshes, nodes and tube length were assessed. The result revealed that HRMECs in the HG group formed more meshes and nodes than the controls; in addition, the tube total length in the HG group was higher than in the control group (Fig. 5B and D). However, after the blocking of ASK1 and p38, the tube meshes, nodes and tube length were reduced compared with 
A

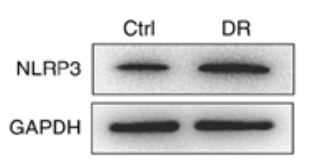

B

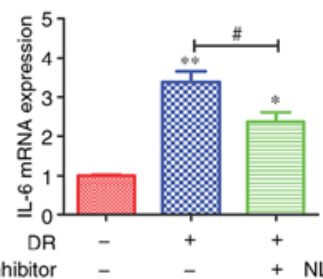
NLRP3 inhibitor
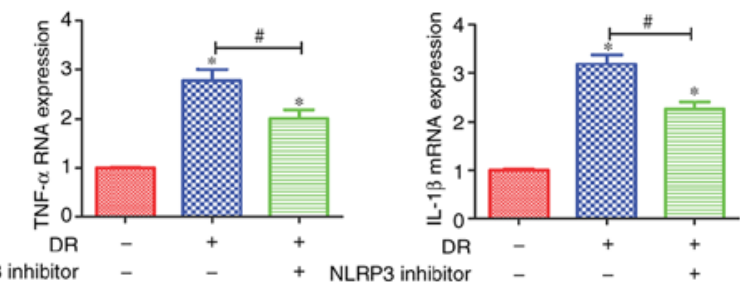

C
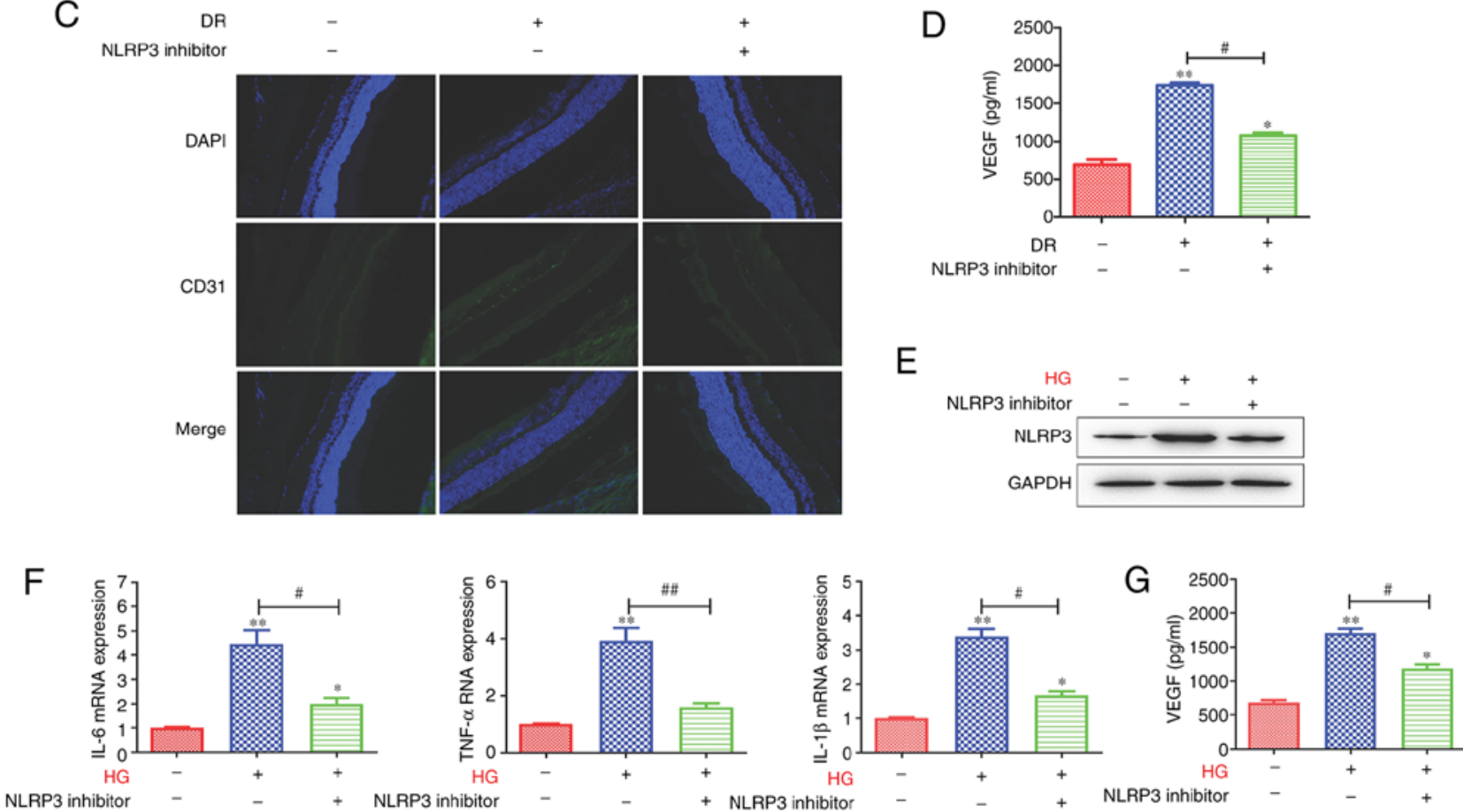

Figure 2. NLRP3-mediated tissue inflammatory response promotes microvascular cell proliferation in the retina. (A) The protein expression level of NLRP3 was increased in the DR groups. (B) Following the of blocking NLRP3, the mRNA expression of IL-6, TNF- $\alpha$ and IL-1 $\beta$ was downregulated. (C) The expression level of the vascular marker, CD31, and (D) the VEGF secretion level were decreased following the inhibition of NLRP3. In addition, in the HRMEC cell model, (E) HG enhanced the protein expression of NLRP3, which was inhibited by NLPR3 inhibitor. (F) The levels of the inflammatory-related cytokines, IL-6, TNF- $\alpha$ and IL-1 $\beta$, were enhanced in the HG-induced HRMEC cell model and were inhibited by the blocking of NLRP3. (G) The VEGF secretion level was enhanced, and was decreased following the inhibition of NLRP3. Data are presented as the mean \pm standard deviation from triplicate wells. ${ }^{*} \mathrm{P}<0.05$ and ${ }^{* *} \mathrm{P}<0.01$ compared with the control; ${ }^{\#} \mathrm{P}<0.05$ and ${ }^{\# \#} \mathrm{P}<0.01$ compared with the relative DR animal model group or HG-induced HRMEC cell group. NLRP3, NLR family pyrin domain containing 3; DR, diabetic retinopathy; IL, interleukin; TNF, tumor necrosis factor; VEGF, vascular endothelial growth factor; HG, high glucose; HRMEC, human retinal microvascular endothelial cell; Ctrl, control.

the HG group (Fig. 5B and D). These data indicated that the ASK1/p38 signaling axis plays a role in the tube formation of retinal microvascular endothelial cells.

Angiogenesis is inhibited by blocking ASK1 and p38. Subsequently, the present study investigated whether ASK1 and p38 mediates angiogenesis. The results revealed that the levels of the angiogenesis-related marker IB4 were markedly increased in the DR model group (Fig. 6); However, IB4 expression was inhibited by the blocking of ASK1 and p38 (Fig. 6). These data suggested that ASK1 and p38 play a role in angiogenesis in DR.

\section{Discussion}

DR is a leading cause of blindness among the working-age population worldwide (22). It is induced by diabetes, which causes the pathological characteristics, such as, retinal capillaries, arterioles and venules, and the subsequent effects of leakage from or occlusion of small vessels (22-24). Laser therapy is the main effective therapy for the preservation of sight in proliferative retinopathy (24); however, it cannot reverse visual loss. Hence, the development of novel and effective therapeutic strategies for DR is imperative.

In the present study, it was revealed that NLRP3 plays an important role in aberrant retinal angiogenesis in DR. It was firstly identified that the ASK1/p38-mediated NLRP3 inflammasome signaling pathway contributed to aberrant retinal angiogenesis in DR. The mRNA expression levels of inflammatory-related cytokines (IL-6, TNF- $\alpha$ and IL-1 $\beta$ ) were upregulated in DR; moreover, the levels of the vascular-related markers, CD31 and VEGF were also increased in DR. After the blocking of NLRP3, ASK1 or p38, the expression levels of inflammatory cytokines were downregulated and those of the vascular-related marker, IB4, were also decreased.

It is known that hyperglycemia can lead to a series of inflammatory mediators in diabetes, which can further activate the hyperinflammatory response and finally damage retinal microvascular cells (25). Chronic inflammation is one of the key mechanisms that are triggered during the pathogenesis 
A

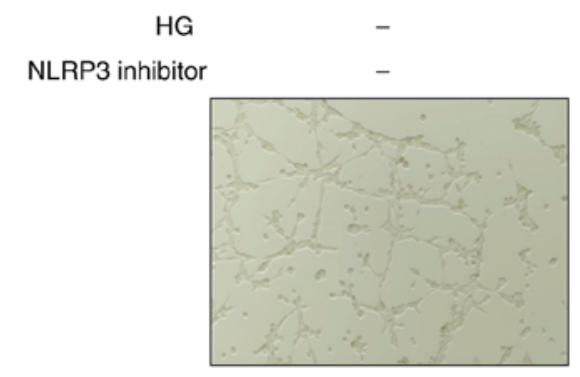

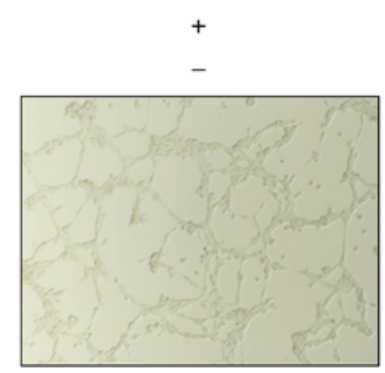

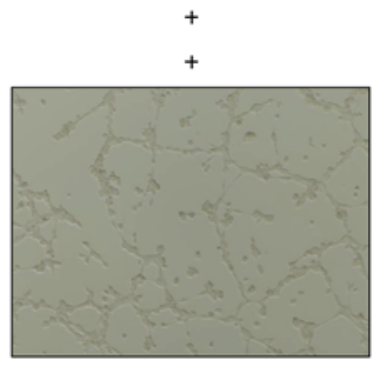

B

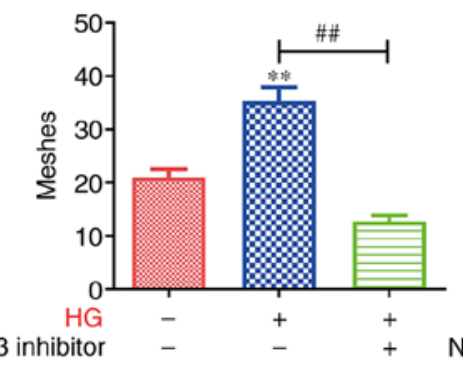

C

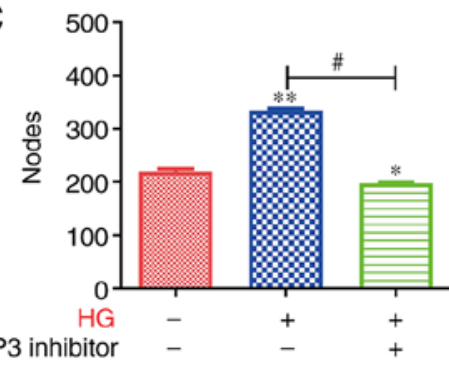

D

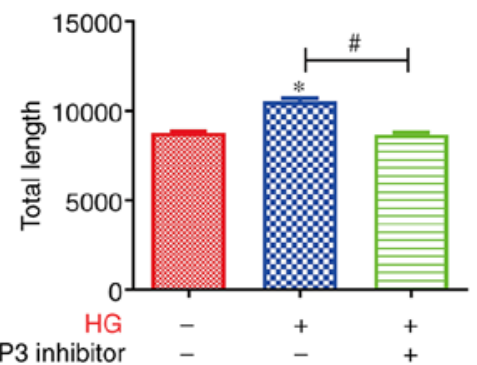

Figure 3. Tube formation of retinal microvascular endothelial cells is inhibited by the blocking of NLRP3. (A) The tube formation was markedly enhanced in the HG group of HRMECs and was inhibited by blocking of NLRP3. Then, the (B) tube meshes, (C) nodes and (D) tube length were counted, and the results revealed that the HRMECs in the HG group formed more meshes and nodes than the control; the tube total length in the HG group was higher than that in the control group. However, after the blocking of NLRP3, the tube meshes, nodes and tube length were reduced compared with the HG group. "P<0.05 and ${ }^{* *} \mathrm{P}<0.01$ compared with the control; ${ }^{*} \mathrm{P}<0.05$ and ${ }^{\# \#} \mathrm{P}<0.01$ compared with the relative HG-induced HRMEC cell group. NLRP3, NLR family pyrin domain containing 3; HG, high glucose; HRMECs, human retinal microvascular endothelial cells.

A
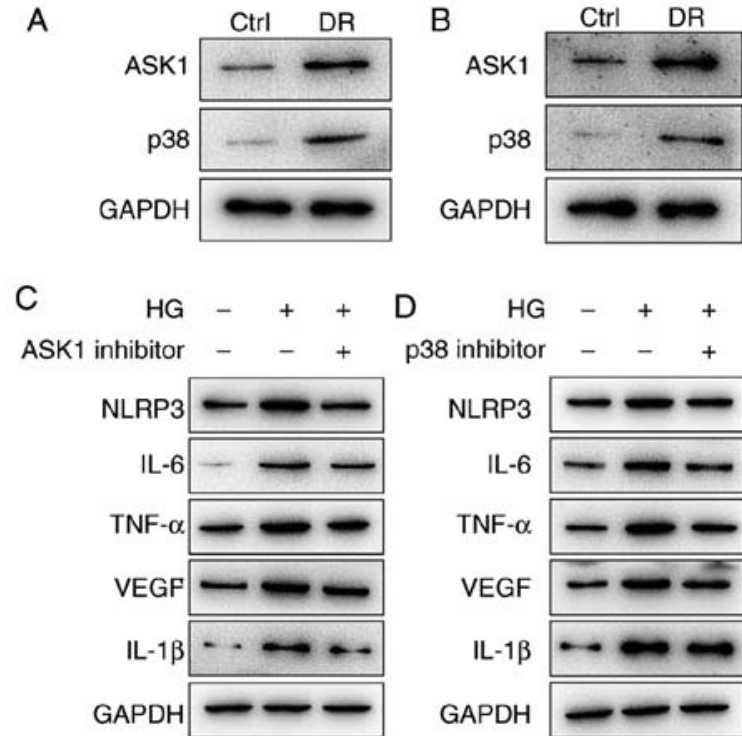

Figure 4. The expression level of inflammasome NLRP3 is upregulated via the activation of the ASK1/p38 signaling axis. (A) The protein expression levels of AKS1 and p38 were upregulated in the DR animal group. (B) The results of the HG-induced HRMEC group were similar to those of the DR animal group. (C) The NLPR3, IL-6, TNF- $\alpha$, IL-1 $\beta$ and VEGF protein expression levels were inhibited by (C) ASK1 inhibitor and (D) p38 inhibitor. NLRP3, NLR family pyrin domain containing 3; ASK1, apoptosis signal-regulating kinase 1; DR, diabetic retinopathy; HG, high glucose; HRMEC, human retinal microvascular endothelial cell; IL, interleukin; TNF, tumor necrosis factor; VEGF, vascular endothelial growth factor; Ctrl, control.

of DR, and the reduction of the inflammation response can alleviate the development and progression of DR (26). Related research has indicated that NLRP3 plays a crucial key in the development of the chronic inflammatory response through

the secretion of related cytokines, such as IL- 6 , TNF- $\alpha$ and IL-1 $\beta$ (27). The activation of NLRP3 contributes to the development and progression of chronic inflammatory diseases (28). In the present study, the results revealed that the NLRP3-mediated tissue inflammatory response promoted microvascular cell proliferation in the retina. In DR animal model, the protein expression of NLRP3 was upregulated in the DR group. The DR group was then treated with NLRP3 inhibitor; following treatment with the inhibitor, the mRNA expression levels of the inflammatory cytokines, IL-6, TNF- $\alpha$ and IL-1 $\beta$ were decreased compared with the DR group without treatment with the inhibitor. In addition, the expression level of CD31 was decreased in the inhibitor treatment group. The VEGF secretion level was also decreased following treatment with the NLRP3 inhibitor. In addition, the NLRP3 protein expression level in HRMECs in the HG group was upregulated, and was suppressed following treatment with the inhibitor. In the HG groups of the HRMECs, the mRNA expression levels of IL- 6 , TNF- $\alpha$ and IL- $1 \beta$ were enhanced compared with the control, and were decreased following treatment with the NLRP3 inhibitor. The secretion levels of VEGF in the cells were similar to those observed in the animal model of DR.

ASK1 is an apoptosis-related protein, which is activated in response to a variety of stress-related stimuli via distinct mechanisms and activates MKK4 and MKK3, which in turn activate JNK and p38 (29). Previous studies have demonstrated that ASK1 can contribute to the development and progression of inflammatory response $(30,31)$. For example, the bacterial infection-engaged inhibition of ASK1 is responsible for regulating Erk1/2- and p38-MAPK activation, but not JNK-MAPK signaling $(31,32)$. It has thus been suggested that ASK1 and p38 have a close association in the inflammatory response. In 
A
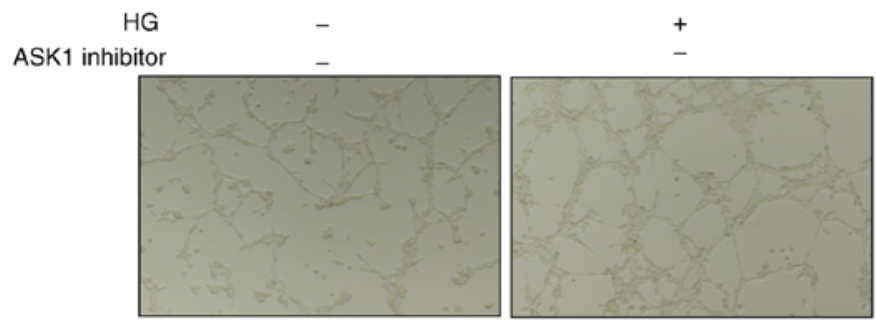

B
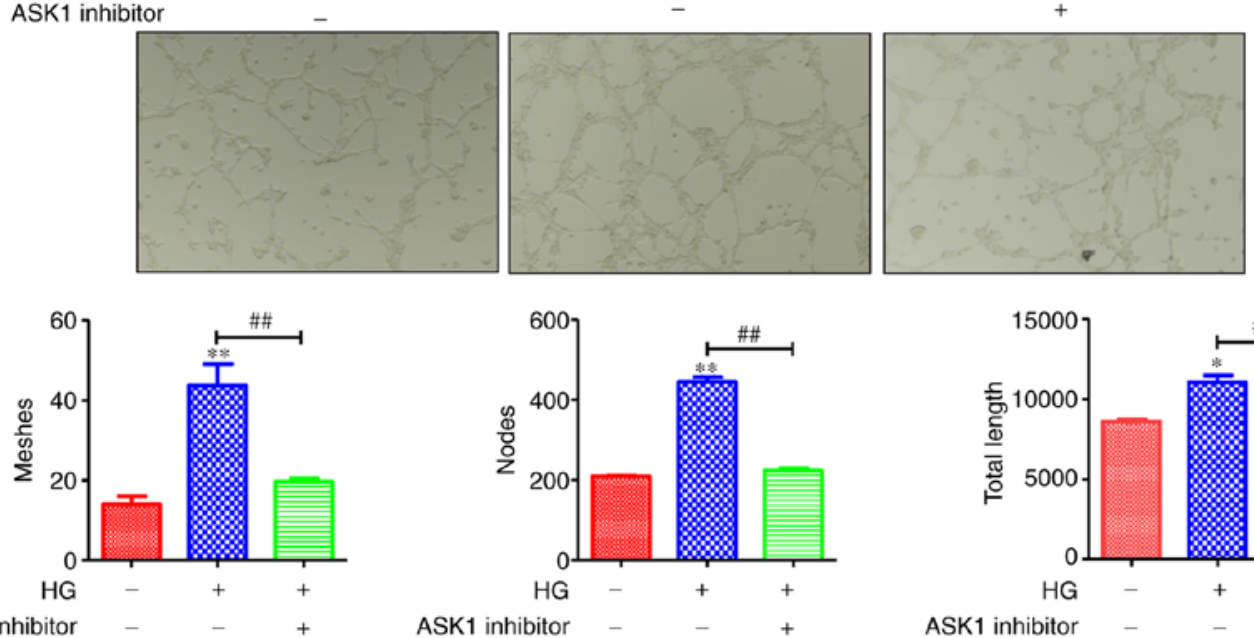

ASK1 inhibitor

ASK1 inhibitor

\section{C}

HG

p38 inhibitor
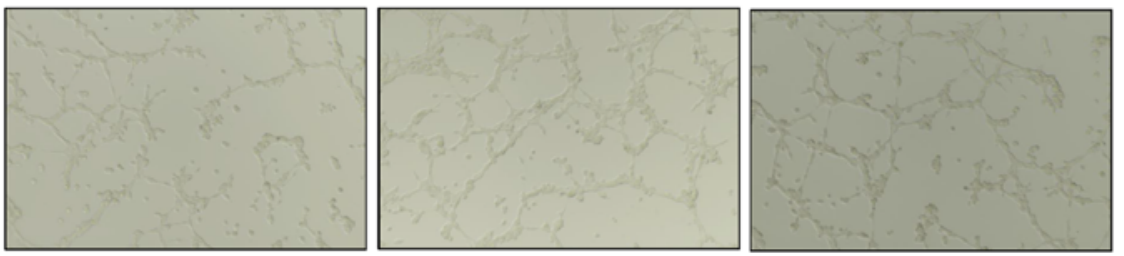

D
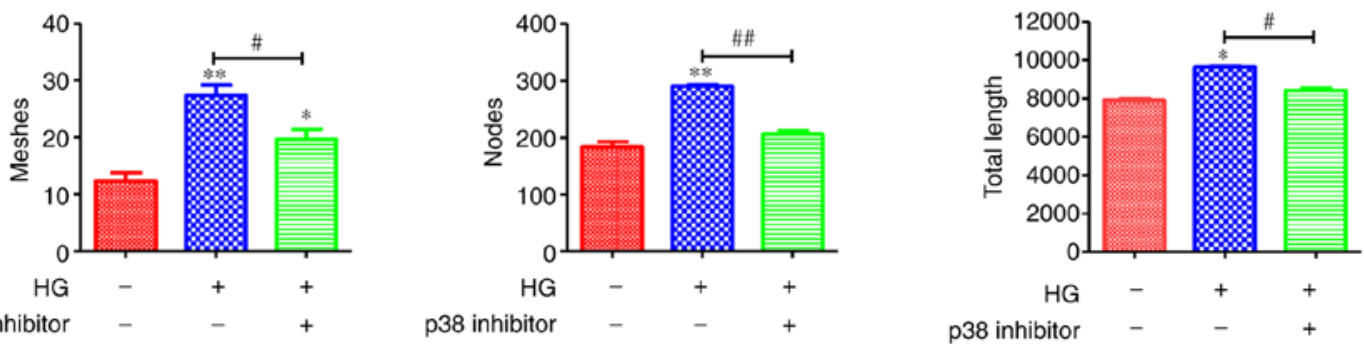

Figure 5. ASK1/p38 signaling axis regulates the tube formation of retinal microvascular endothelial cells. (A) The tube formation was markedly enhanced in the HG group of the HRMECs and was inhibited by blocking of ASK1. Then, the (B) tube meshes, nodes and tube length were counted, and the results revealed that the HRMECs in the HG group formed more meshes and nodes than the control; in addition, the tube total length in the HG group was higher than that of the control group. However, after blocking of ASK1, the tube meshes, nodes and tube length were reduced compared with the HG group. In addition, (C) the tube formation was inhibited by blocking of p38 and the (D) tube meshes, nodes and tube length were counted; the results revealed that the blocking of p38 inhibited the tube formation. ${ }^{*} \mathrm{P}<0.05$ and ${ }^{* *} \mathrm{P}<0.01$ compared with the control; ${ }^{\#} \mathrm{P}<0.05$ and ${ }^{\# \#} \mathrm{P}<0.01$ compared with the relative HG-induced HRMEC cell group. ASK1, apoptosis signal-regulating kinase 1; HG, high glucose; HRMECs, human retinal microvascular endothelial cells.

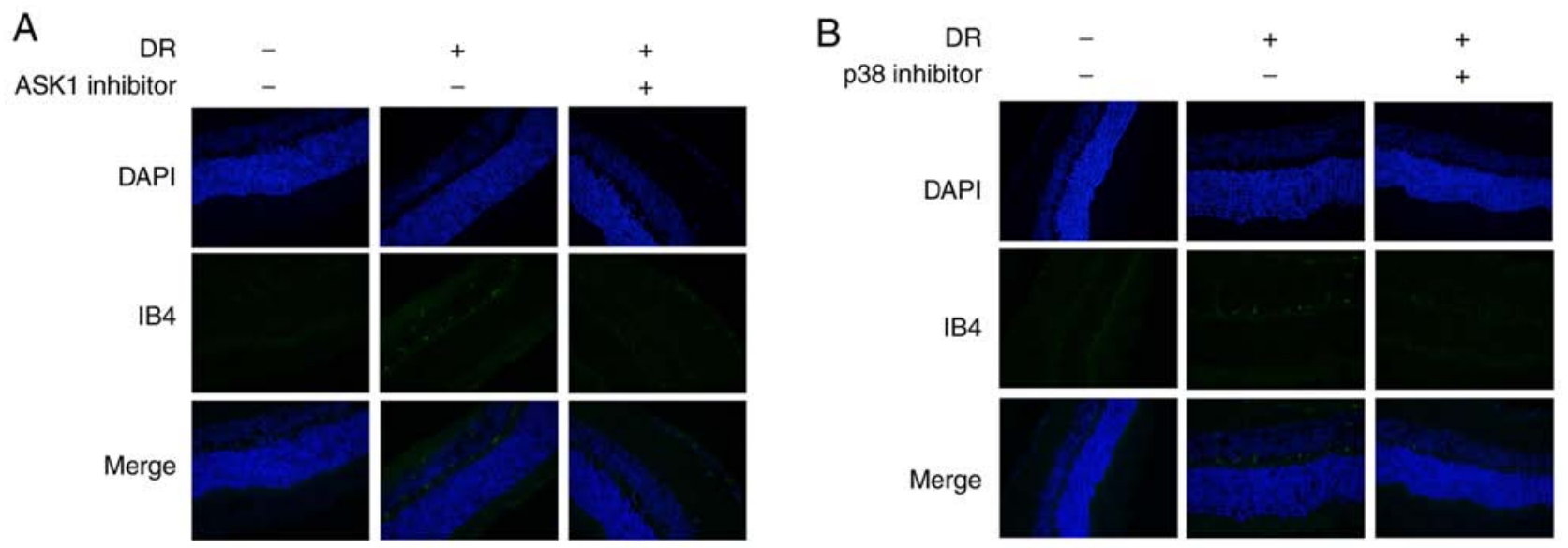

Figure 6. Angiogenesis is inhibited by the blocking of ASK1 and p38. The levels of the angiogenesis-related marker, IB4, were obviously increased in the HG model group; however, IB4 expression was inhibited after the blocking of (A) ASK1 and (B) p38. ASK1, apoptosis signal-regulating kinase 1; HG, high glucose. 
addition, some drugs can protect the retinal photoreceptor cells via the activation of the $\mathrm{p}$-Erk1/2/Nrf2/Trx/ASK1 signaling pathway in diabetic mice (33). It has been suggested that ASK1 plays an important role in diabetic-related diseases (33). In the present study, the data indicated that the expression level of inflammasome NLRP3 was upregulated through the activation of the ASK1/p38 signaling axis; moreover, the ASK $1 / \mathrm{p} 38$ signaling axis contributed to the tube formation of retinal microvascular endothelial cells and development of angiogenesis in DR. It was revealed that ASK1 and p38 protein expression levels were upregulated in DR; however, following the blocking of ASK1 and p38, the protein expression levels of NLRP3 and related cytokines (IL-6, TNF- $\alpha$ and IL-1 $\beta$ ) were downregulated. In addition, the tube formation of retinal microvascular endothelial cells was inhibited by the blocking ASK1 and p38. These data revealed that ASK1 and p38 mediated the NLRP3 inflammasome signaling pathway contributing to aberrant retinal angiogenesis in DR.

In conclusion, the present study demonstrated that DR induced inflammatory response and microvascular cell proliferation. NLRP3 contributed to DR-mediated inflammatory development and progression, which promoted the inflammatory-related cytokine expression. Moreover, DR promoted the tube formation of retinal microvascular endothelial cells and angiogenesis. Further research revealed that NLRP3-mediated aberrant retinal angiogenesis in DR was regulated by ASK1 and $\mathrm{p} 38$.

\section{Acknowledgements}

Not applicable.

\section{Funding}

This research was financially supported by National Natural Science Foundation of China (grant no. 81700852), the Young Talent's Subsidy Project in Science and Education of the Department of Public Health of Jiangsu Province (grant no. QNRC2016140), the Social Development Project of Jiangsu Provincial Science and Technology Department (grant no. BE2017627) and the Funded Project of the Wuxi Municipal Health and Family Planning Commission (grant no. Q201623).

\section{Availability of data and materials}

The datasets used during the present study are available from the corresponding author upon reasonable request.

\section{Authors' contributions}

WZ and ZZ conceived and designed the study. ZZ, SL, and YP performed the experiments. WZ,SL, LC, XH and ND collected, analyzed and interpreted the data. WZ and ZW drafted and critically revised the study for important intellectual content. All authors read and approved the final submission.

\section{Ethics approval and consent to participate}

All of the animal experiments were performed in accordance with the Guidelines for the Care and Use of Laboratory
Animals published by the US National Institutes of Health (NIH Publication no. 85-23, revised 1996), and were approved by the Experimental Animal Ethics Committee of the Affiliated Wuxi No. 2 People's Hospital of Nanjing Medical University (Nanjing, China).

\section{Patient consent for publication}

Not applicable.

\section{Competing interests}

The authors declare that they have no competing interests.

\section{References}

1. Congdon NG, Friedman DS and Lietman T: Important causes of visual impairment in the world today. Jama 290: 2057-2060, 2003.

2. Laiginhas R, Madeira C, Lopes M, Neves JN, Barbosa M, Rosas V, Carvalho D, Falcão-Reis F and Falco M: Risk factors for prevalent diabetic retinopathy and proliferative diabetic retinopathy in type 1 diabetes. Endocrine 66: 201-209, 2019.

3. Dong C, Liu P, Wang H, Dong M, Li G and Li Y: Ginsenoside $\mathrm{Rb} 1$ attenuates diabetic retinopathy in streptozotocin-induced diabetic rats1. Acta Cir Bras 34: e201900201, 2019.

4. Jeganathan VS, Wang JJ and Wong TY: Ocular associations of diabetes other than diabetic retinopathy. Diabetes Care 31: 1905-1912, 2008

5. Dehdashtian E, Mehrzadi S, Yousefi B, Hosseinzadeh A, Reiter RJ, Safa M, Ghaznavi H and Naseripour M: Diabetic retinopathy pathogenesis and the ameliorating effects of melatonin; involvement of autophagy, inflammation and oxidative stress. Life Sci 193: 20-33, 2018.

6. Abougalambou SS and Abougalambou AS: Risk factors associated with diabetic retinopathy among type 2 diabetes patients at teaching hospital in Malaysia. Diabetes Metab Syndr 9: 98-103, 2015.

7. Rubsam A, Parikh S and Fort PE: Role of inflammation in diabetic retinopathy. Int J Mol Sci 19: 942, 2018.

8. Wang H, Wang G, Liang Y, Du X, Boor PJ, Sun J, Khan MF: Redox regulation of hepatic NLRP3 inflammasome activation and immune dysregulation in trichloroethene-mediated autoimmunity. Free Rad Biol Med 143: 223-231, 2019.

9. Chen H, Zhang X, Liao N, Mi L, Peng Y, Liu B, Zhang S and Wen F: Enhanced expression of NLRP3 inflammasome-related inflammation in diabetic retinopathy. Invest Ophthalmol Vis Sci 59: 978-985, 2018

10. Chaurasia SS, Lim RR, Parikh BH, Wey YS, Tun BB, Wong TY, Luu CD, Agrawal R, Ghosh A, Mortellaro A, et al: The NLRP3 inflammasome may contribute to pathologic neovascularization in the advanced stages of diabetic retinopathy. Sci Rep 8: 2847 , 2018.

11. Guigui S, Lifshitz T and Levy J: Diabetic retinopathy in Africa: Advantages of screening. Postgrad Med 123: 119-125, 2011.

12. Nomura K, Lee M, Banks C, Lee G and Morris BJ: An ASK1-p38 signalling pathway mediates hydrogen peroxide-induced toxicity in NG108-15 neuronal cells. Neurosci Lett 549: 163-167, 2013.

13. Sobhan PK, Zhai Q, Green LC, Hansford LM and Funa K: ASK1 regulates the survival of neuroblastoma cells by interacting with TLX and stabilizing HIF-1 $\alpha$. Cell Signal 30: 104-117, 2017.

14. Katome T, Namekata K, Guo X, Semba K, Kittaka D, Kawamura K, Kimura A, Harada C, Ichijo H, Mitamura Y and Harada T: Inhibition of ASK1-p38 pathway prevents neural cell death following optic nerve injury. Cell Death Differ 20: 270-280, 2013.

15. Flaumenhaft R: Stressed platelets ASK1 for a MAPK. Blood 129: 1066-1068, 2017.

16. Huang C, Zhu HJ, Li H, Li QX, Li FM, Cheng L and Liu YG: P38-MAPK pathway is activated in retinopathy of microvascular disease of STZ-induced diabetic rat model. Eur Rev Med Pharmacol Sci 22: 5789-5796, 2018.

17. Fortin J, Patenaude A, Deschesnes RG, Côté MF, Petitclerc E and C-Gaudreault R: ASK1-P38 pathway is important for anoikis induced by microtubule-targeting aryl chloroethylureas. J Pharm Pharm Sci 13: 175-190, 2010. 
18. Barros-Miñones L, Orejana L, Goñi-Allo B, Suquía V, Hervías I, Aguirre N and Puerta E: Modulation of the ASK1-MKK3/6-p38/MAPK signalling pathway mediates sildenafil protection against chemical hypoxia caused by malonate. Br J Pharmacol 168: 1820-1834, 2013.

19. Liu W, Gu J, Qi J, Zeng XN, Ji J, Chen ZZ and Sun XL: Lentinan exerts synergistic apoptotic effects with paclitaxel in A549 cells via activating ROS-TXNIP-NLRP3 inflammasome. J Cell Mol Med 19: 1949-1955, 2015

20. Wang N, Zhang C, Xu Y,Li S, Tan HY, Xia W and Feng Y: OMICs approaches-assisted identification of macrophages-derived MIP- $1 \gamma$ as the therapeutic target of botanical products TNTL in diabetic retinopathy. Cell Commun Signal 17: 81, 2019.

21. Mengual L and Olivan M: Quantitative RNA analysis from urine using real time PCR. Methods Mol Biol 1655: 227-237, 2018.

22. Klein R, Lee KE, Knudtson MD, Gangnon RE and Klein BE: Changes in visual impairment prevalence by period of diagnosis of diabetes: The wisconsin epidemiologic study of diabetic retinopathy. Ophthalmology 116: 1937-1942, 2009.

23. Wilkinson CP, Ferris FL III, Klein RE, Lee PP, Agardh CD Davis M,Dills D, Kampik A, Pararajasegaram R and Verdaguer JT; Global Diabetic Retinopathy Project Group: Proposed international clinical diabetic retinopathy and diabetic macular edema disease severity scales. Ophthalmology 110: 1677-1682, 2003.

24. Kang EY, Chen TH, Garg SJ, Sun CC, Kang JH, Wu WC, Hung MJ, Lai CC, Cherng WJ and Hwang YS: Association of statin therapy with prevention of vision-threatening diabetic retinopathy. JAMA Ophthalmol 137: 363-371, 2019.

25. Lowry F: Compound could help diabetic patients walk tightrope between heperglycemia, hypoglycemia. CMAJ 154: 705-707, 1996

26. Li S, Yang H and Chen X: Protective effects of sulforaphane on diabetic retinopathy: Activation of the nrf2 pathway and inhibition of NLRP3 inflammasome formation. Exp Anim 68: 221-231, 2019.
27. Fouad AA, Abdel-Aziz AM and Hamouda AA: Diacerein downregulates NLRP3/Caspase-1/IL-1 $\beta$ and IL-6/STAT3 pathways of inflammation and apoptosis in a rat model of cadmium testicular toxicity. Biol Trace Elem Res 195: 499-505, 2019.

28. Tartey S and Kanneganti TD: Differential role of the NLRP3 inflammasome in infection and tumorigenesis. Immunology 156: 329-338, 2019.

29. Ju A, Cho YC, Kim BR, Park SG, Kim JH, Kim K, Lee J, Park BC and Cho S: Scaffold role of DUSP22 in ASK1-MKK7-JNK signaling pathway. PLoS One 11: e0164259, 2016.

30. Iriyama T, Takeda K, Nakamura H, Morimoto Y, Kuroiwa T, Mizukami J, Umeda T, Noguchi T, Naguro I, Nishitoh H, et al: ASK1 and ASK2 differentially regulate the counteracting roles of apoptosis and inflammation in tumorigenesis. EMBO $\mathrm{J} 28$ : 843-853, 2009.

31. Yang D, Liu X, Xu W, Gu Z, Yang C, Zhang L, Tan J, Zheng X, Wang Z, Quan S, et al: The edwardsiella piscicida thioredoxin-like protein inhibits ASK1-MAPKs signaling cascades to promote pathogenesis during infection. PLoS Pathog 15: e1007917, 2019.

32. Gong X, Duan Y, Zheng J, Wang Y, Wang G, Norgren S and Hei TK: Nephroprotective effects of n-acetylcysteine amide against contrast-induced nephropathy through upregulating thioredoxin-1, Inhibiting ASK1/p38MAPK pathway, and suppressing oxidative stress and apoptosis in rats. Oxid Med Cell Longev 2016: 8715185, 2016.

33. Ren X, Sun H, Zhang C, Li C, Wang J, Shen J, Yu D and Kong L: Protective function of pyridoxamine on retinal photoreceptor cells via activation of the $\mathrm{pErk} 1 / 2 / \mathrm{Nrf} 2 / \mathrm{Tr} x / \mathrm{ASK} 1$ signalling pathway in diabetic mice. Mol Med Rep 14: 420-424, 2016.

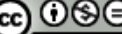

This work is licensed under a Creative Commons Attribution-NonCommercial-NoDerivatives 4.0 International (CC BY-NC-ND 4.0) License. 